\title{
X-ray Study on the Formation of Nano-crystallite and Amorphous Phase of Mo-Fe by Ball Milling
}

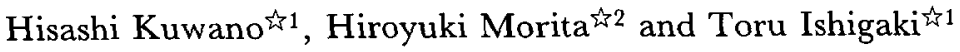 \\ t1 Muroran Institute of Technology, 27-1 Mizumoto-cho, Muroran 050. \\ of Technology, [Present Address: Mitsumi Electric Co., Ltd., 1601 Sakai, Atsugi 243].
}

Received January 13, 1995

\section{SYNOPSIS}

Pure Mo powders are mechanically attrited with a hardened steel vial and balls in an $\mathrm{Ar}$ atmosphere to produce nanostructural and amorphous Mo-Fe alloys. A time evolution of the solid state amorphization caused by the attrition is classified into three stages. In the initial stage the crystallite size of Mo decreases down to $10 \mathrm{~nm}$ together with the increase in the lattice strain up to $1.8 \%$. Plastic deformation is a dominating cause of the crystallite size reduction. In the next stage, the crystallite size decreases down to a minimum dimension of $5 \mathrm{~nm}$. The reduciton of the grain size is accompanied by a decrease in the lattice strain and the solid solutioning of the Fe impurities which are introduced from the vail and balls in the prior stage. The grain size reduction in this stage is interpreted in terms of recovery process of the stored strain. In the final stage where the Fe content in the Mo lattice saturates about $18 \mathrm{at} \%$, a transition from the nano-crystallites to an amorphous phase takes place.

KEY WORDS

nanocrystallite, amorphous phase, grain size, ball milling, Mo-Fe,

\section{Introduction}

Ball milling is a distinguished technique to produce nanoscaled grains and amorphous phases in solid state(1,2). The process usually accompanies an increase in the lattice strain ${ }^{(3)}$, reduction in the crystallite size ${ }^{(1,3)}$, and mixing of elemental powders in the atomic scale ${ }^{(4)}$. The lattice strain increased in the short time milling followed by a decrease in the longer milling times ${ }^{(3,5)}$. A correlation between the lattice strain and the reduction of the crystallite size has not been clearly understood.

Ball milling of $\mathrm{Cr}-18.5 \% \mathrm{Fe}$ in an argon atmosphere yielded a nanocrystallite of $3 \mathrm{~nm}$ grain size after 96 hours milling, but the formation of the amorphous phase has not been confirmed ${ }^{(6)}$. On the other hand, ball milling of $\mathrm{Cr} 7 \mathrm{OFe} 30$ powders in a nitrogen atmosphere finally yielded amorphous phases containing 15.1 at $\%$ nitrogen content $(7)$. These facts suggest that the solutioning of nitrogen atom plays an important role to yield amorphous phase since atomic size difference between $\mathrm{Fe}$ and $\mathrm{Cr}$ is quite small. In case of Mo-Fe, however, a mixure of Mo and Fe mixed in ratios of $4: 6$ to $6: 4$ yielded amorphous phases after the $500 \mathrm{~h}$ ball milling in an argon atmosphere ${ }^{(8)}$. In this case, a large difference of the atomic size between Mo and $\mathrm{Fe}$ seems to be meaningful for the amorphous formation.

In the present study, Mo powders are mechanically attrited in an argon atmosphere to produce nanostructures and Mo-Fe amorphous phases. Fe is intended to be mixed from the vial and ball materials by abrasion. Processes of straining, mixing and alloying of $\mathrm{Fe}$, reduction of the crystallite size, and formation of amorphous phase are investigated to clearify the solid state amorphization.
II Experimental procedure

Mo powders of $99.9 \%$ purity were mechanically milled by steel balls in a steel vial both of which are made of SUJ2 steel in an Ar atmosphere using a high enrgy type SPEX $8000 \mathrm{mixer} / \mathrm{mill}$. Nominal chemical compositions of the SUJ2 steel are $1.0 \% \mathrm{C}$, $0.75 \% \mathrm{Mn}, 0.25 \% \mathrm{Si}, 1.5 \% \mathrm{Cr}$ and the balanced Fe. A powder-to-ball weight ratio was 1 to 8 . X-ray diffraction $(\mathrm{XRD})$ patterns of $\mathrm{Cu}-\mathrm{K} \alpha$ radiation were decomposed into $K \alpha 1$ and $K \alpha 2$ component by using a profile-fitting program. The $\mathrm{X}$-ray profiles were analysed as a superposition of the crystalline and the amorphous component for the long term milling. Broadening of the X-ray diffraction peaks was interpreted in terms of the reduction of the grain size $D$ and the increase in the lattice stain $\varepsilon$. Mo and Fe contents in the milled powders were analyzed by a Xray energy dispersive spectrometer (EDS).

\section{Results}

\subsection{Mixing and alloying of $\mathrm{Fe}$}

As shown in Fig.1, XRD peaks due to Mo become broader with increasing milling time. Their peak positions shift to higher angles with the milling time becomes longer than $10 \mathrm{~h}$. After the $470 \mathrm{~h}$ milling, sharp diffraction peaks characteristic of the crystalline Mo are entirely replaced by halo peaks which are ascribed to an amorphous phase. Since a (110) diffraction peak of $\mathrm{Fe}$ appears about $45^{\circ}$ for $\mathrm{t}=5$ and $25 \mathrm{~h}, \mathrm{Fe}$ impurities which originate from the abrasion of the vial and balls are mixed with Mo powders. However, the $(110)_{\mathrm{Fe}}$ peak disappears when the milling time becomes longer than $80 \mathrm{~h}$, which means that the Fe impurities from the vial and balls dissolve to make solid solutions with Mo. 

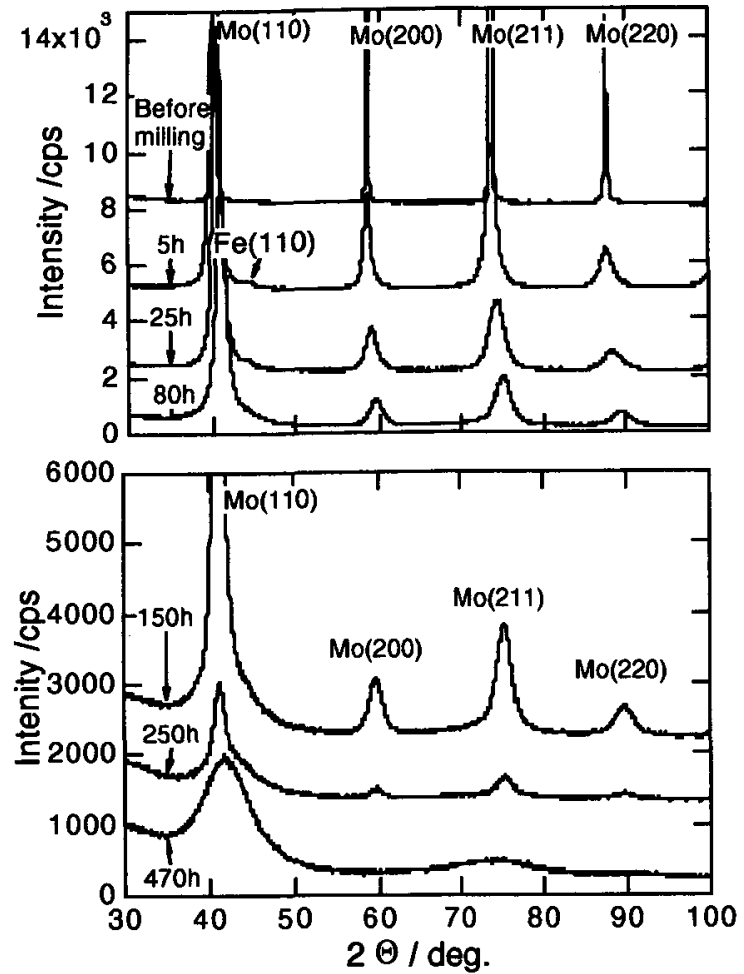

Fig.1 Change in the X-ray diffraction profiles of Mo after high energy ball-milling with the milling time labelled(CuK $\alpha$ radiation).

The lattice parameter $a$ of Mo changes in three stages with milling time as shown in Fig.2. In the first stage for $k 15 \mathrm{~h}$, there is no appreciable change in $a$ although (110)Fe diffraction peak coexists with diffraction peaks of Mo. So the iron impurities introduced from the vial and balls exist as a mechanical mixture. In the second stage between $t=15 \mathrm{~h}$ and $100 \mathrm{~h}$, a gradual decrease in $a$ occurs. The change is ascribed to the alloying of $\mathrm{Fe}$ impurities into Mo because the total iron content measured by EDS increases as mentioned below. In the third stage for $\mathrm{t}>100 \mathrm{~h}$, no appreciable change in $a$ occurs.

Open circles in Fig.3 present the Fe content measured by EDS as a function of milling time. The Fe content increases with the milling time becomes longer. It is reasonable to consider that the decrease in $a$ of Mo mentioned above is caused by the alloying of $\mathrm{Fe}$ into the Mo lattice. Hence the $\mathrm{Fe}$ content dissolved in the Mo lattice may be estimated from $a$ on the basis of Vegard's raw. As shown by solid circles in Fig. 3, the Fe content determined by XRD increases with an increase of the milling time after an elapse of the duration time of about $10 \mathrm{~h}$, and saturates to a value of 18 at $\%$ for $t>100 h$. Since halo peaks characteristic of the amorphous phases appears about $80 \mathrm{~h}$, we may consider that the saturated iron content is a critical one where a transition from the nanostructure to the amorphous phase takes place.
Large discrepancies between the results of XRD and those of EDS may be explained as follows. Fe atoms may exist as the finely dispersed iron mixtures, solutes in the nanostructured Mo, those in the amorphous phase, and chemical segregations at grain boundaries. EDS measurements can detect a sum of these $\mathrm{Fe}$ atoms in different situations. However in case of XRD measurements, only $\mathrm{Fe}$ atoms substituted in the bcc Mo lattice are detected to result in the lower Fe content than EDS.

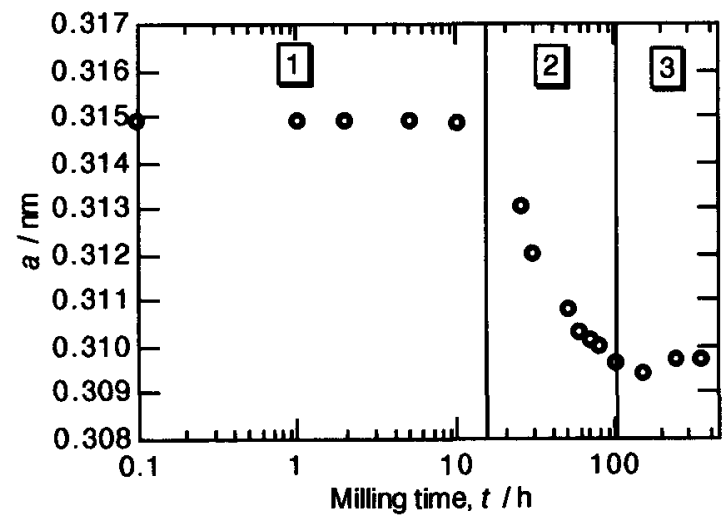

Fig.2 Lattice parameter of Mo as a function of milling time.

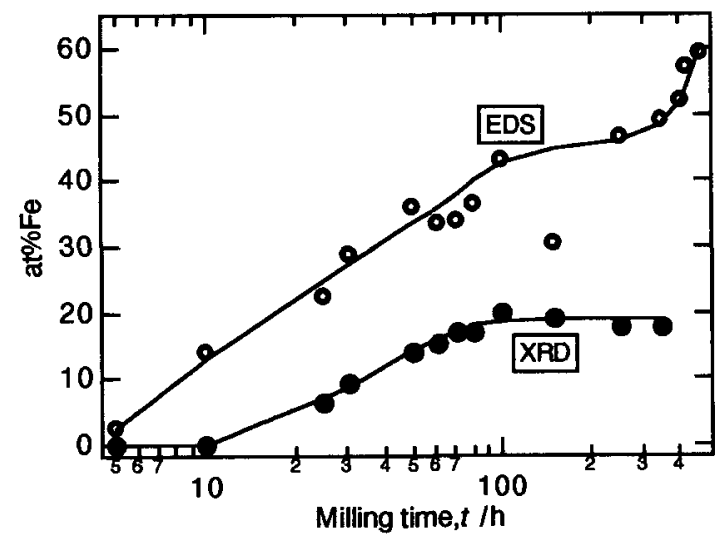

Fig.3 Iron content of Mo measured by energy dispersive spectroscopy (EDS) and X-ray diffraction (XRD) as a function of milling time.

\subsection{Formation of the nano-crystallite}

The widths of the X-ray diffraction peaks due to Mo become broader with the milling time becomes longer. In general two contributions are responsible for the line broadening. One is a decrease in the crystallite size $D$, and the other is an increase in the lattice strain $\varepsilon$. These two contributions are separated using the following relation;

$$
B \cos (\Theta / \lambda)=1 / D+2 \varepsilon \sin (\Theta \lambda)
$$

where $B, \lambda$ are the integrated line width and the wave length, respectively. A typical example of the plot for 


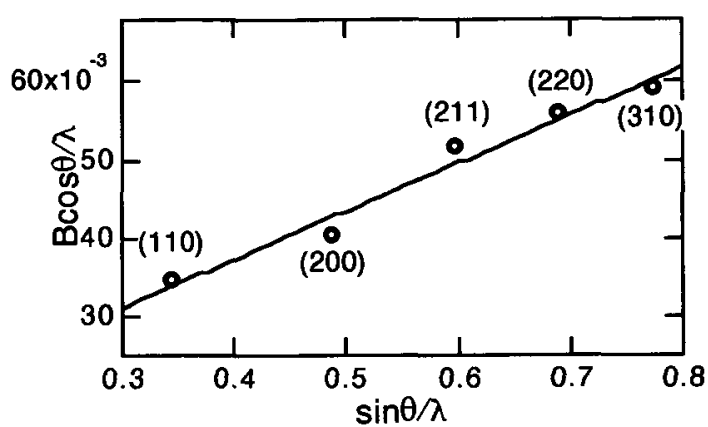

Fig.4 $B \cos \Theta / \lambda$ versus $\sin \Theta / \lambda$ plot for Mo diffraction lines after ball milling for 10 hours.

eq.(1) is given for the $10 \mathrm{~h}$ milling in Fig.4. A good linear relationship is confirmed among five data as shown by a solid line. Deviations of the experimental points from the line are not strongly dependent on the diffraction plane. This result suggests that $\varepsilon$ is nearly isotropic. $D$ and $\varepsilon$ are determined from the $y$ intersection and the slope of the line obtained by a least-square-fitting according to eq.(1), respectively. Values of $D$ obtained in such a way are plotted versus milling time in Fig.5. In the first ten hours, $D$ decreases rapidly down to about $10 \mathrm{~nm}$, and then gradually approaches to a constant value of $5 \mathrm{~nm}$. The size is a little larger than $3 \mathrm{~nm}$ for the $\mathrm{Cr}-\mathrm{Fe}$ nanocrystallite ${ }^{6}$ ). Further longer ball-milling causes no appreciable change in $D$.

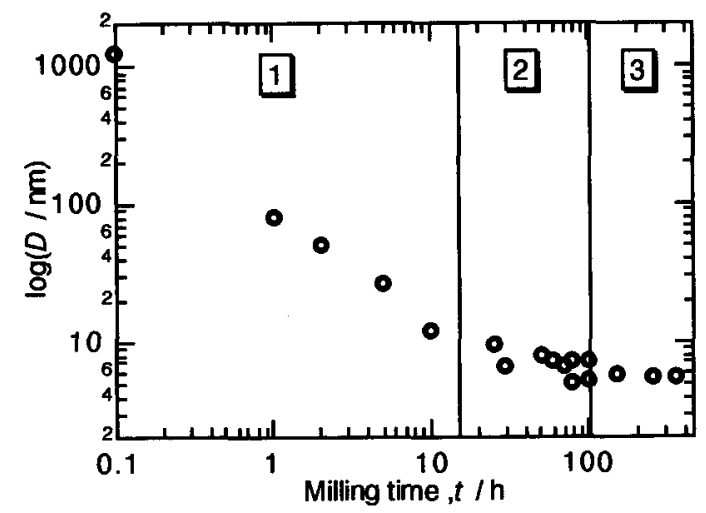

Fig.5 Crystallite size of Mo measured from the Xray diffraction line broadening as a function of milling time.

As shown in Fig.6, $\varepsilon$ changes in three stages in a similar way with $a$. At first, $\varepsilon$ increases to a maximum of $1.8 \%$ with increasing milling time, then decreases in the milling time periods of $20 \mathrm{~h}<\mathrm{t}<100 \mathrm{~h}$. Though the scattering of the experimental data is a little large, $\varepsilon$ has a tendency to approach to a constant of ca. $1.4 \%$ in the final stage. In Fig.7, $\varepsilon$ is plotted versus $D$. Since the reduction in $D$ down to $10 \mathrm{~nm}$ is accompanied by the increase in $\varepsilon$ in the first stage of the ball milling, a plastic deformation of Mo caused by the ball colllsions may be a controlling process of this stage. But the following reduction in $D$ occurs with the decrease of $\varepsilon$, indicating that a controlling mechanism is different from one mentioned above. It is well known that recovery during thermal annealing envolves a reduction of $\varepsilon$ introduced by cold work.

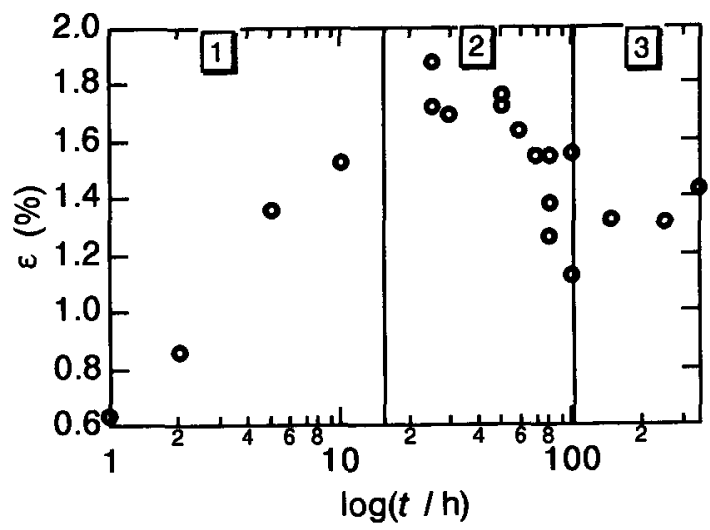

Fig.6 Strain of Mo induced by high-energy ball milling as a function of milling time.

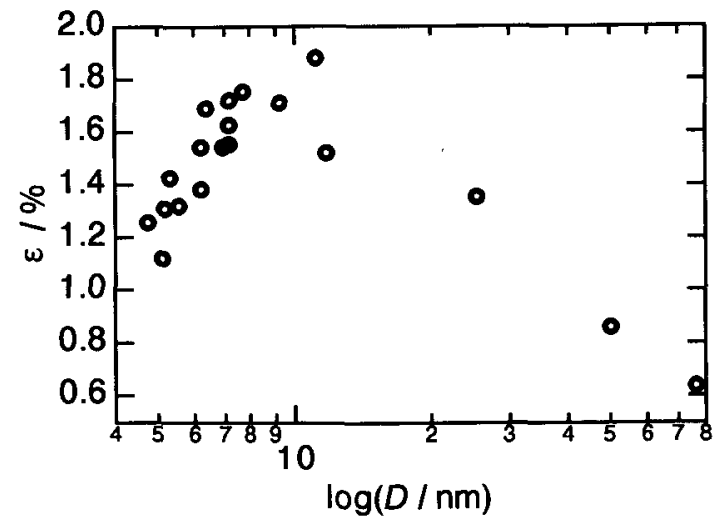

Fig.7 Strain as a function of crystallite size of Mo ball milled for various times.

In order to confirm whether the reduction of $\varepsilon$ and $D$ are cooperative in the recovery process during thermal annealing of nano-structured phases, a ballmilled sample with $D=7 \mathrm{~nm}$ has been annealed at $400^{\circ} \mathrm{C}$ for various times. The $400^{\circ} \mathrm{C}$ annealing causes a negligible small change in the lattice parameter as shown in Fig.8(a), which means no appreciable change in the Fe content. However the annealing is accompanied by a reduction in $D$ from 7 to $5 \mathrm{~nm}$ together with a decrease in $\varepsilon$. in a similar way of the second stage of the ball-milling. The process may be interpreted in terms of recovery which usually envolves a rearrangement of dislocations and annihilation of point defects. 


\subsection{Formation of the amorphous phase}

The bottom of the (110) Mo diffraction peak swells gradually around $2 \Theta=41$ degree when the milling time becomes longer than $80 \mathrm{~h}$, as typically confirmed in the profile of the $250 \mathrm{~h}$ milling in Fig.1. After the $470 \mathrm{~h}$ milling, the swelling grows to a halo peak to replace the (110) Mo peak. The XRD peaks due to the
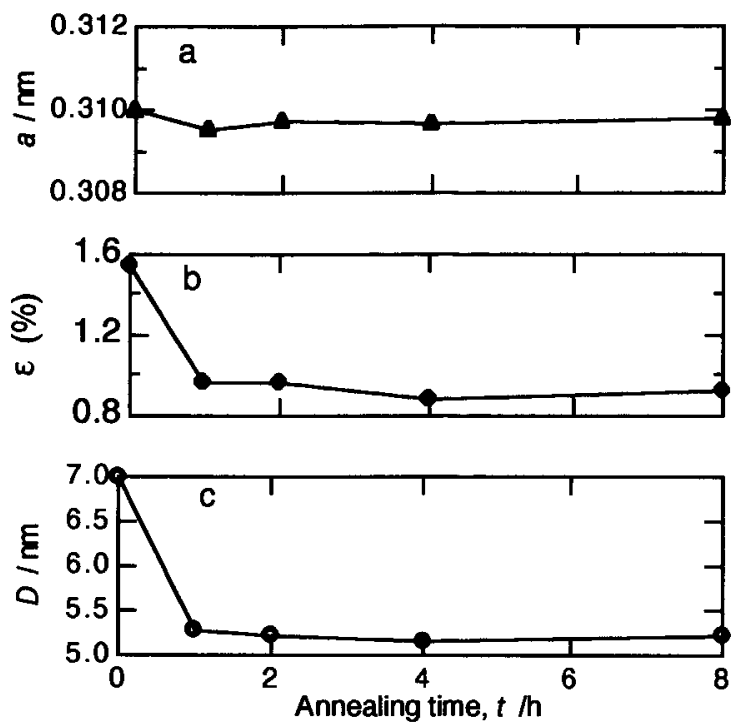

Fig.8 Change in the lattice parameter(a), strain(b) and crystallite size(c) of the nano-sized Mo during annealing at $400^{\circ} \mathrm{C}$.

crystalline Mo-Fe are replaced entirely by two broad peaks around 41 and 75 degree of two-theta angle. These XRD pattern changes are interpreted in terms of a phase transition from the nanocrystallite to an amorphous phase. The transition occurs when $D$ and the Fe content become about $5 \mathrm{~nm}$ and 18at\%, respectively.

\section{Discussion}

According to Fecht( ${ }^{(3)}$, three different stages were defined in the ball milling process of hcp Ru and $\mathrm{CsCl}$-type AlRu. We can also confirm three stages in the ball milling process of bcc Mo. In the initial stage less than 15 hours of milling time, the crystallite size reduces down to $10 \mathrm{~nm}$. Since strain increases up to $1.8 \%$, the reduction in the crystallite size is mainly caused by plastic deformation which introduces the fragmentaion of crystalline grains due to tangled dislocation networks. The crystallite size of $10 \mathrm{~nm}$ may be a critical dimension to which the grain size reduction can be attained by the plastic deformation, because finer the crystallite size becomes higher the yield strength of Mo does.

An annealing experiment for ball-milled powders has revealed that the crystallite size of the nanophase reduces with a decrease in the stored strain. A similar phenomenon is expected to occur during ball milling. We anticipate that an instantaneous temperaure rise due to the collison of the high energy balls would give rise to recovery process in the second stage of ball milling, in which a further reduction in the crystallite size from 10 to $5 \mathrm{~nm}$ occurs. The rearrangement of dislocations to form stable arrays such as low-angle boundaries or subboundaries may be responsible for the grain size reduction. According to an equilibrium phase diagram (9), the solubility of $\mathrm{Fe}$ in $\mathrm{Mo}$ is expected to be negligibly small below $1000 \mathrm{~K}$. However nanosized Mo prepared by ball milling can dissolve $\mathrm{Fe}$ to a maximum amount of $18 \mathrm{at} \%$. A transition from the nanophase to the amorphous phase takes place when the $\mathrm{Fe}$ contnet in Mo exceeds the solubility limit. The distortion stored by the solute solutioning plays a role to induce the transiton.

\section{Conclusion}

Solid state amorphization of Mo caused by the high energy ball-milling is defined in three stages.

1 In the initial stage, the crystallite size reduces down to $10 \mathrm{~nm}$ due to the plastic deformation, accompanied by the increase in the lattice strain. $\mathrm{Fe}$ impurities introduced from the vial and balls are mechanically mixd with Mo powders during the stage.

2 In the following stage, further reduction of the crystallite size down to a minimum value of $5 \mathrm{~nm}$ occurs together with a decrease in the lattice strain. Spontaneous recovery is responsible for the process. Fe mixtures makes solid solutions with Mo to a saturation content of $18 \mathrm{at} \%$. 3 A transiton from the nanocrystallite to the amorphous phase occurs finally when the $\mathrm{Fe}$ content in the nanocrystalline Mo lattice reaches to a critical value and the crystallite size becomes about $5 \mathrm{~nm}$.

\section{Acknowledgement}

The authous would like to express deep thanks to Iketani Science and Technology Fundation for the financial support .

\section{References}

1) H.J.Fecht, E.Hellstern, Z.Fu and W.L.Johnson: Advances in Powder Metallurgy, 1 -3, (1989),111. 2) Ricardo B. Schwarz: Appl.Phys. Lett. 49(3), (1986), 146.

3) H.J.Fecht: Nanostructured Materials, 1,(1992), 125.

4) B.Fultz, G.Le Caer and P.Matteazzi: J.Mater.

Res., 4,(1989), 1450.

5) H.Kuwno and Y.Hamaguchi: JSPM, 39,(1992), 211

6) H.Kuwano, H.Ouyang and B.Fultz:

Nanostructured Materials, $1,(1992), 143$.

7) T.Fukunaga,E.Ishikawa, N.Kuroda, C.H.Lee and U.Mizutani: Proc.4th Japan-Russia Sympo. on Mechanochemistry(1992) 69.

8) Junji Kuyama, Keiichi N. Ishihara and

P.H.Shingu:JSPM, 38,(1991),910.

9) O.Kubaschewski: Iron-Binary Phase Diagrams, Springer-Verlag Berlin Heiderberg New York, (1982),p65. 